Pure and Applied Mathematics Quarterly

Volume 1, Number 3

(Special Issue: In Memory of

Armand Borel, Part 2 of 3 )

$665-682,2005$

\title{
On Modular Functors and the Ideal Teichmüller Tower
}

\author{
Po $\mathrm{Hu}$ and Igor Kriz
}

\section{INTRODUCTION}

The purpose of this note is to connect certain results of Hatcher, Lochak and Schneps [7] on the Teichmüller tower of mapping class groups with the language of mathematical conformal field theory introduced by Segal [19] as recently made rigorous by the authors ([8], [9], see also [5]). The results of [7] realize a part of Grothendieck's Esquisse d'un programme ([6]), further explained by Drinfeld [4]. In the present note, we interpret this result in the framework of $[8,9,5]$. In a certain form, these results are very close to Bakalov and Kirillov [1, 2]. Yet, the present note gives a somewhat different perspective. In our formalism, modular functors and conformal field theories are (lax) morphisms of certain structures which we call lax commutative monoids with cancellation. Our main point is to study these structures along with the morphisms. This tidies up the story considerably.

The program $[6]$ proposed characterizing the absolute Galois group $\operatorname{Gal}(\overline{\mathbb{Q}} / \mathbb{Q})$ by its action on the pro-finite completion of the fundamental group of $\mathbb{P}^{1}-$ $\{0,1, \infty\}$, by using the extension making up its étale fundamental group. This actually allows the embedding of a large subgroup of $G a l(\overline{\mathbb{Q}} / \mathbb{Q})$ into the group of automorphism of the profinite completion $\hat{F}_{2}$ of the free group on two generators, and by generalizing $\mathbb{P}^{1}-\{0,1, \infty\}$ to more general varieties, notably moduli spaces of curves with punctures, one may write down a number of relations which the Galois subgroup must satisfy. The main open question of the program is whether these relations completely characterize the Galois group.

Received February 27, 2005.

The first author was supported by NSF grant DMS 0303505, the second author was supported by NSF grant DMS 0305853 . 
For technical reasons (which can be eliminated by refining the theory), HatcherLochak-Schneps [7] actually consider the subgroup $G=\operatorname{Gal}(\overline{\mathbb{Q}} / K)$ where $K$ is the extension of $\mathbb{Q}$ by adjoining all roots of 1 and 2 . In the paper [7], they show that this group $G$ acts, through a group $\Lambda$ of automorphisms of $\hat{F}_{2}$ characterized by specific equations, on all the profinitely-completed mapping class groups of Riemann surfaces with punctures and boundary components. They further show that the action is compatible with inclusions of surfaces and call the resulting structure a Teichmüller tower. In a remarkable philosophical discussion in their introduction, the authors of [7] mention that perhaps an even stronger structure on the mapping class groups should exist, which they call the ideal Teichmüller tower. They propose that their group $\Lambda$ should act on that hypothetical structure as well.

It has been noticed that another area of mathematics, namely conformal field theory, supplies a structure which is a candidate for the ideal Teichmüller tower. In this note, we show that the Hatcher-Lochak-Schneps group $\Lambda$ in fact acts on that structure when properly axiomatized. Implicitly or explicitly, this connection was certainly noted in [4], and perhaps even more explicitly in [3]. In fact, the connection starts with the work of Moore and Seiberg [13], who prove, in a physical setting, Verlinde's conjecture [21]. To do this, they intuitively computed the group of constraints of modular functors, or conformal blocks, in conformal field theory and showed that those constraints implied the Verlinde conjecture. It was in fact later noticed that the Moore-Seiberg constraints correspond closely to the constraints of groups such as $\Lambda$ (in fact, the correspondence can be made exact by adjusting minor technical details of definitions). Bakalov and Kirillov $[1,2]$ treat much of the Moore-Seiberg theory mathematically, and make the connection with the language of [7]. The main point of this note is to show that in our approach to conformal field theory, this kind of connection is almost immediate.

What exactly, in mathematical terms, is conformal field theory? In physics, conformal field theory is a quantum field theory with a particular kind of Lagrangian on a 2-dimensional manifold called a worldsheet. The main idea of string theory is to reinterpret this as a kind of quantum mechanics (not field theory) of loops (strings), which show up as boundary components of worldsheets. Worldsheets are then, in a way, analogues of Feynman diagrams for strings, which however have many peculiar properties different from Feynman diagrams (notably smoothness and additional symmetries) which we will not discuss here. The conformal structure, which is a complex structure on the worldsheet, comes from a gauge-theoretical approach to string theory, which makes it easier to quantize (the gauge is the complex structure). 
Now this motivation leads to a specific mathematical structure on the set of all possible worldsheets, which was first observed by Segal [19]. In fact, he noticed that worldsheets must be compact complex manifolds with real-analytically parametrized boundary components (this is what he calls rigged surfaces). The salient structure on these surfaces which string theory uses are: groupoid structure (more precisely stack over the site of analytic manifolds and open covers), disjoint union, and gluing. Both the notions of conformal field theory and modular functor (which, in physical terms, is the gauge anomaly of conformal field theory) can be axiomatized by means of that structure.

Therefore, consulting mathematical physics suggests that the structure outlined by Segal should, indeed, be the 'ideal Teichmüller tower'. There is in principle no difficulty with defining that structure rigorously, it is routine. However, there are technical difficulties which lead to 2-category theory. A rigorous definition of the structure, which is called stack of lax commutative monoids with cancellation (SLCMC) was given by the authors in [8], [9], see also Fiore [5] for important categorical preliminaries. If we are interested merely in mapping class groups, the situation somewhat simplifies and one encounters a lax commutative monoid with cancellation (LCMC) which we call $\mathcal{C}_{\text {top }}$ and which is the main subject of this note.

The present note is organized as follows. In Section 2, we briefly review the notions of SLCMC, and modular functors. Precise definitions are recorded in Section 4 (the Appendix). In Section 3, we state and prove the main theorem which says that the Hatcher-Lochak-Schneps group $\Lambda$ acts on the profinite completion of $\mathcal{C}_{t o p}$, and hence on modular functors with a finiteness condition. We conclude that section with a few speculations and open problems.

Acknowledgement: We would like to thank Leila Schneps for conversations which motivated this note, and Bojko Bakalov and Sasha Kirillov for historical remarks.

\section{LAX COMMUTATIVE MONOIDS WITH CANCELATIONS OF RIGGED SURFACES}

Segal [19] defines rigged surfaces as 2-dimensional compact manifolds with complex structure and with all boundary components $c$ parametrized by real-analytic diffeomorphisms $S^{1} \rightarrow c$. We shall consider two groupoids with objects rigged surfaces: $\mathcal{C}$ has as morphisms holomorphic diffeomorphisms preserving boundary component parametrizations (although not necessarily the order of boundary components). $\mathcal{C}_{\text {top }}$ has as morphisms isotopy classes (with boundary fixed during the isotopies) of diffeomorphisms (not necessarily holomorphic) preserving 
boundary component parametrizations (although, again, not necessarily the order of boundary components). As shown in [8], $\mathcal{C}$ is, in addition, naturally a stack over the site of complex analytic manifolds with the topology of open covers. (One can also consider $\mathcal{C}_{\text {top }}$ as a stack, but it is equivalent just to the disjoint union of the classifying stacks of the mapping class groups.)

Now we may ask what additional interesting structure there is on $\mathcal{C}, \mathcal{C}_{\text {top }}$. Segal [19] identified two operations interesting from the point of view of conformal field theory: disjoint union and gluing. The meaning of disjoint union is obvious, to understand gluing, note that rigged surfaces come with a canonical orientation induced by the complex structure, which in turn lets us consistently distinguish two orientations of boundary component parametrizations: we call those inbound and outbound (the exact choice of which is which is immaterial here). Now however if we have a rigged surface $X$ with an inbound boundary component $c$ and outbound boundary component $d$, we can glue $c$ to $d$ (using the boundary parametrizations), thus obtaining another rigged surface which is usually denoted by $\check{X}$ (although that notation does not reflect the dependence on $c, d$ ).

The exact properties satisfied by the operations of disjoint union and gluing are not easy to encode rigorously, and such rigorization was not given in [19]. It is done in [8], see also [9]. To make this note self-contained, we review this boring rigorous definition in the Appendix. The resulting structure is called a stack of lax commutative monoids with cancelations (SLCMC). The main point is that gluing along two pairs is not dependent on the order of gluing, and is also distributive under disjoint union, while disjoint union is commutative associative and unital. Of course, all these relations are only true up to coherence isomorphisms, and all the fuss is about finding a general abstract principle by which one should choose coherence diagrams to be satisfied by those coherence isomorphisms. This is also done in [8], and this is what the word lax refers to. Also, all the operations are compatible with stack structure in a suitable sense. Remark: Fiore [5] points out that by conventions of category theory, the word lax should be replaced by the prefix pseudo-. However, we do not adopt this terminological convention here, as the word lax seems more intuitive, while 'pseudo-' sounds awkward.

Now SLCMC's, as any lax algebraic structure, form a 2-category, where morphisms are those commuting with the operations up to natural coherence isomorphisms satisfying suitable properties, most notably compatibility, in the obvious sense, with coherence isomorphisms of the structures involved (for most detail, see [5] and the Appendix). We call such morphisms lax morphisms, (in [5] they are called pseudomorphisms). 
Thus, we obtain a lax morphism of SLCMC's

$$
\pi: \mathcal{C} \rightarrow \mathcal{C}_{\text {top }}
$$

Note, again, that on $\mathcal{C}_{\text {top }}$, the stack structure is unimportant, since this is only a disjoint union of classifying stacks of the mapping class groups. Thus, when referring to $\mathcal{C}_{\text {top }}$, we will just talk about an LCMC. One additional variation of the LCMC $\mathcal{C}_{\text {top }}$ will be denoted by $\mathcal{C}_{\text {top }}^{p}$, and in fact we shall have a lax map of LCMC's

$$
p: \mathcal{C}_{\text {top }}^{p} \rightarrow \mathcal{C}_{\text {top }}
$$

which in fact shall be an equivalence of categories. To this end, the objects of $\mathcal{C}_{\text {top }}^{p}$ are rigged surfaces $X$ with a given pants decomposition $P=P_{X}$. By a pants decomposition we mean a collection of closed (unoriented) Jordan curves in $X$ which cut $X$ into pairs of pants. The boundary components are required to be elements of $P_{X}$. Two pants decompositions $P, P^{\prime}$ are considered identical if there is a diffeomorphism $X \rightarrow X$ isotopic to the identity rel the boundary which takes $P$ to $P^{\prime}$. Of course, if $X$ is of genus 0 with $\leq 2$ boundary components, or in genus 1 with 0 boundary components, no pants decomposition is possible. In the genus 0 case with $\leq 2$ boundary components, we consider $P$ vacuous data, but in genus 1 with 0 boundary components, it is useful to consider $P$ a choice of one non-separating closed Jordan curve in $X$ (again, up to isotopy). Now we simply let the morphisms of $\mathcal{C}_{\text {top }}^{p}$ be pulled back from morphisms in $\mathcal{C}_{\text {top }}$ by $p$, so of course we get equivalent categories. It is easily seen how pants decompositions behave under gluing, so $\mathcal{C}_{\text {top }}^{p}$ inherits the structure of an LCMC.

Now there is one more variation which is relevant. Recall that profinite completion $\hat{G}$ of a group $G$ is functorial in groups and homomorphisms, so by completing the isotropy groups of an LCMC $\mathcal{D}$ which is a groupoid, we obtain another LCMC $\widehat{\mathcal{D}}$, which we call the profinite completion of $\mathcal{D}$. Profinite completion preserves equivalence of groupoids. We shall consider the profinite completions $\widehat{\mathcal{C}}_{\text {top }}, \widehat{\mathcal{C}}_{\text {top }}^{p}$ here.

We next need to briefly review modular functors. This concept has been outlined (and the term coined) by Segal [19]. Rigorous definitions, following different approaches, have been given in [20], [1] and [9]. We follow here the approach of [9], which treats modular functors as lax morphisms of SLCMC's. The main point is to introduce another SLCMC $C(\mathcal{M})$ where $\mathcal{M}$ is a set of labels. Classically, this is indeed the same as a finite set $A$, but in [9] we see that it is convenient to think of $\mathcal{M}$ as a 2 -vector space, which is a free finite lax module over the lax semiring $\mathbb{C}_{2}$ of finite-dimensional vector spaces over $\mathbb{C}$ with direct sum and tensor product as operations (without saying more, this is not really much of a generalization, since such $\mathcal{M}$ is required to have a basis set $A$, which remains pretty much rigid under lax automorphisms; speculations can be made toward obtaining a much 
larger category by group-completing $\mathbb{C}_{2}$ in some way, but we won't go into that here). In any case, we shall only describe the LCMC structure on $C(\mathcal{M})$ here; for more details, see [9]. The full subcategory on objects of $C(\mathcal{M})$ with $i$ inbound and $j$ outbound boundary components is

$$
\mathcal{M}^{* \otimes i} \otimes \mathcal{M}^{\otimes j} .
$$

Here $\mathcal{M}^{*}$ is the dual 2-vector space, and the tensor product is the lax tensor product over $\mathbb{C}_{2}$ (with the label approach, we may simply ignore all of this nonsense, and describe the basis of (3), which is the obvious thing, namely $\left.A^{\times(i+j)}\right)$. Now the operation of disjoint union is given by tensor product, and the operation of gluing by the canonical trace map $\operatorname{tr}: \mathcal{M}^{*} \otimes \mathcal{M} \rightarrow \mathbb{C}_{2}$ (which is again elementary if we work in terms of basis).

A finite topological modular functor with labels $\mathcal{M}$ is a lax morphism of LCMC's

$$
\widehat{C}_{\text {top }} \rightarrow C(\mathcal{M}) .
$$

(The notion of topological modular functor is defined in the same way, but dropping the hat. To drop the word 'topological', replace $\mathcal{C}_{\text {top }}$ by $\mathcal{C}$ and LCMC by SLCMC.)

It is traditional to add the assumption that the value of the modular functor on a disk is non-trivial only for one label, denoted by 1 . We shall assume that in this paper.

Conjecture 1. All topological modular functors are finite.

All examples we know satisfy this conjecture. One more thing is worth commenting on. In this setting, the adjective "topological" is not as severe a restriction on a modular functor as it may seem. A modular functor, when restricted to the semigroup of annuli, specifies a central extension of the group of positively oriented real-analytic diffeomorphisms $S^{1} \rightarrow S^{1}$ by $\mathbb{C}^{\times}$. Such central extensions have an invariant called central charge. From the results of [10], there follows

Theorem 2. The central charge of a modular functor is always a rational number. This number is an integer if the modular functor takes values in 1-dimensional vector spaces only. Moreover, a modular functor is topological (laxly factors through $\mathcal{C}_{\text {top }}$ ) if and only if its central charge is 0.

Essentially, the rationality and integrality statements about central charge are proved by showing that there negation produces a contradiction with the gluing axiom. (Non-integrality for higher-dimensional modular functors is possible, an example is given by higher level chiral WZW models.) For the last statement, the main point is that the central charge captures all local behaviour of a modular 
functor, i.e. a modular functor with central charge 0 is locally constant on the moduli stack $\mathcal{C}$. Now in [10], it is proved (following again earlier outlines of Segal [19] and Quillen [15]) that

Proposition 3. There exists a modular functor Det ${ }^{\otimes 2}$ with 1 label (hence $\otimes$ invertible) and central charge -4 .

(The reason Det does not work is that it introduces super-vector spaces and hence does not satisfy our definition of modular functor as stated; see [10] and [9] for more explanation.)

\section{The ACTION of $\Lambda$}

In [7], Hatcher, Lochak and Schneps define a group $\Lambda$ as follows. Consider the commutator subgroup $\widehat{F}_{2}^{\prime}$ of the profinite completion $\widehat{F}_{2}$ of the free group on two generators $F_{2}$. Then $\Lambda$ is the set of elements $f \in \widehat{F}_{2}^{\prime}$ such that $x \mapsto x, y \mapsto f^{-1} y f$ extends to an automorphism $F_{f}$ of $\widehat{F}_{2}$ which satisfies the following relations. For elements $a, b$ in any profinite group $G$, let $f(a, b)$ be the element which is the image of $f$ under $x \mapsto a, y \mapsto b$. There are four relations. The first three are

$$
\begin{gathered}
f(x, y) f(y, x)=1, \\
f\left((x y)^{-1}, x\right) f\left(y,(x y)^{-1}\right) f(x, y)=1, \\
f\left(x_{34}, x_{45}\right) f\left(x_{15}, x_{12}\right) f\left(x_{23}, x_{34}\right) f\left(x_{45}, x_{15}\right) f\left(x_{12} x_{23}\right)=1 .
\end{gathered}
$$

The relation (6) takes place in the profinite completion of the mapping class group of a rigged surface of genus 0 with 5 boundary components $c_{1}, \ldots, c_{5}$ and $x_{i j}$ are curves separating $c_{i}, c_{j}$ from the remaining boundary components every two of which have geometric intersection number 2 if they share a subscript, and geometric intersection 0 otherwise, cf. [7]. The fourth (last) relation of the definition of $\Lambda$ takes place in the mapping class group of a rigged surface of genus 1 with 2 boundary components. Recall this is isomorphic to a quotient of the Artin Braid group $B_{4}$ with standard generators $\sigma_{1}, \sigma_{2}, \sigma_{3}$ (with relations $\left.\sigma_{i} \sigma_{i+1} \sigma_{i}=\sigma_{i+1} \sigma_{i} \sigma_{i+1}\right)$, modulo its center which is generated by $\left(\sigma_{1} \sigma_{2} \sigma_{3}\right)^{4}$. Let (see [17], also [14, 12, 7]) $e_{1}=\left(\sigma_{1} \sigma_{2}\right)^{6}, e_{2}=\sigma_{3} \sigma_{2} \sigma_{1} \sigma_{2}^{-1} \sigma_{3}^{-1}, e_{3}=\left(\sigma_{2} \sigma_{3}\right)^{6}$. Then the relation is

$$
f\left(e_{3}, \sigma_{1}\right) f\left(\sigma_{2}^{2}, \sigma_{3}^{2}\right) f\left(e_{2}, e_{3}\right) f\left(e_{1}, e_{2}\right) f\left(\sigma_{1}^{2}, \sigma_{2}^{2}\right) f\left(\sigma_{3}, e_{1}\right)=1 .
$$

A trivial, but likely helpful remark to the novice: Dehn twists do not depend on orientation. Suppose we are twisting around a closed Jordan curve $c$ in a rigged surface $X$. Give $c$ an orientation. Then $c$ cuts its tubular neighborhood into two halfs, which the orientation distinguishes as "right" and "left" (distinguished by whether $c$ is outbound or inbound on the rigged surface $X^{\prime}$ obtained from $X$ by cutting along $c$ ). We can then make the convention, say, that our Dehn twist 
means we cut $X$ along $c$ and glue back, after changing the parametrization of the "left" copy of $c$ by multiplying by $e^{2 \pi i t}, t \in[0,1]$ (this defines a loop in the moduli space, more precisely stack). Now suppose we reverse the orientation of $c$, and keep the definition of Dehn twist. Then the direction in which we rotate the left copy of $c$ before reattaching reverses, but so does right and left; therefore, we are rotating the other copy of $c$ in the opposite direction, obtaining the same twist. This makes it possible to forget orientations of the curves along which we are twisting in formulas such as (4) - (7).

We are now ready to state our main result, which is a reinterpretation of Theorem B of [7] in terms of the SLCMC $\mathcal{C}_{\text {top }}^{p}$. We introduce two more notions: Recall the lax map of SLCMC's (2). A morphism $f: X \rightarrow Y$ is said to preserve pants decomposition if $p(f)$ maps the pants decomposition $P_{X}$ to the pants decomposition $P_{Y}$ (in the obvious sense). On the other hand, $f$ is said to be a change of pants decomposition if $X=Y$ as rigged surfaces (but do not necessarily have the same pants decomposition) and $f=I d$ as a map. It is well known ([7]) that the subcategory of changes of pants decompositions is generated by two types of morphisms, called $A$-move and $S$-move. The $A$-move occurs in two adjacent pairs of pants with a curve $c$ in common, and the $S$-move occurs in one pair of pants where the curve $c$ is attached to itself. In both cases, the curve $c$ is replaced by another curve, which constitutes the change of pants decomposition. To describe the other curve ([7]) here, we note that the $A$-move can be characterized as replacing $\left(\sigma_{1}\right)^{2}$ be $\left(\sigma_{2}\right)^{2}$ in the pure braid group $\tilde{B}_{3}$, which is identified in the standard way with the mapping class group of a genus 0 rigged surface with four boundary component in the case of the $A$ move, while the $S$-move can be characterized as replacing $\sigma_{1}$ by $\sigma_{2}$ in $B_{3}$, which is identified in the standard way with the mapping class group of a genus 1 rigged surface with one boundary component.

Theorem 4. The group $\Lambda$ acts on the $L C M C \widehat{\mathcal{C}}_{\text {top }}^{p}$ by maps of $L C M C$ 's which are strict in the sense that they have identical coherences. The action is defined as follows. Let $f \in \Lambda$. First of all, $f$ acts trivially on objects. $f$ also acts trivially on morphisms preserving pants decomposition. On pants decomposition changes, the action of $f$ is described by its effect on A-moves and $S$-moves as follows. Suppose an $A$-move or $S$-move $\phi: X \rightarrow Y$ replaces a curve $b$ in the pants decomposition $P_{X}$ by a curve $c$. Then

$$
f(\phi)=f\left(b^{\epsilon}, c^{\epsilon}\right) \circ \phi
$$

where $\epsilon=\epsilon_{\phi}$ is 1 if $\phi$ is an $A$-move and 2 if $\phi$ is an $S$-move.

Remark: Note that the order of composition doesn't matter in (8), as $f\left(b^{\epsilon}, c^{\epsilon}\right)$ commutes with $\phi$ since $p(\phi)=I d$. Even more precisely, this is accurate only if 
we realize that when we write $f\left(b^{\epsilon}, c^{\epsilon}\right)$ before or after $\phi$, it represents different morphisms in $\mathcal{C}_{\text {top }}^{p}$ (automorphisms of different objects distinguished only by pants decompositions); of course, $p$ maps these two morphisms to the same element of the mapping class group of the same surface.

Remark: It is remarked in [7] (Introduction) that, as a corollary of the results of [14], [12], the group $\Lambda$ contains canonically the absolute Galois group of the extension $K$ of $\mathbb{Q}$ obtained by adding all the $n$-th roots of unity and 2 for all $n \geq 1$. This is, in fact, the motivation for considering such groups, outlined by Grothendieck [6] and explained by Drinfeld [4]. L. Schneps recently proposed [18] a refinement of the group $\Lambda$ which contains the whole absolute Galois group of $\mathbb{Q}$. The results of [7] (and this note) may be extended to that group using the same methods, but the notion of pants decomposition must be replaced by a more refined notion of seamed pants decomposition also outlined in [18].

Proof of Theorem 4: Using the ideas of [7], this is quite easy. First, we show that $\Lambda$ acts consistently on the groupoid $\mathcal{C}_{\text {top }}^{p}$. To this end, first consider the subcategory $\mathcal{D}_{1}$ of $\mathcal{C}_{\text {top }}^{p}$ with the same set of objects and morphisms given by pants decomposition changes. Showing that $\Lambda$ acts consistently on $\mathcal{D}_{1}$ follows directly from Theorem 2 of [7]. To summarize briefly, the theorem says that the 2-dimensional $\mathrm{CW}$ complex $H T$ obtained by taking pants decomposition on a surface $X$ as vertices, $S$-moves and $A$-moves as edges and 2-cells corresponding to the relations (5)-(7) is simply connected. This means that (8) extends by composition consistently to the category $\mathcal{D}_{1}$, as writing a morphism in $\mathcal{D}_{1}$ in two different ways as product of $A$-moves and $S$-moves gives the same result. (The relation (4) is used to ensure that a composing an $A$-move or an $S$-move with its inverse gives indeed the identity.)

Now consider the subcategory $\mathcal{D}_{2}$ of $\mathcal{C}_{\text {top }}^{p}$ on the same objects, with morphisms given by mapping classes preserving pants decompositions. Then $\Lambda$ of course acts consistently on $\mathcal{D}_{2}$, namely by the identity.

Now however the groupoid $\mathcal{C}_{t o p}^{p}$ can be describe as a semidirect product of the groupoid $\mathcal{D}_{1}$ with the groupoid $\mathcal{D}_{2}$. This means that $\mathcal{C}_{\text {top }}^{p}$ is generated by $\mathcal{D}_{1}$ and $\mathcal{D}_{2}$ freely subject to the relation

$$
\phi \circ \psi=\psi^{\phi} \circ \phi
$$

where $\phi: X \rightarrow Y$ is a morphism in $\mathcal{D}_{2}$ (i.e. preserving pants decomposition), $\psi$ is a change of pants decomposition on $X$, and $\psi^{\phi}$ is a change of pants decomposition on $Y$ induced from $\psi$ by $\phi$ (by mapping the pants decomposition bodily).

Therefore, we are done if we can show that the action of $f \in \Lambda$ preserves the relation (9), i.e. that

$$
\phi \circ f(\psi)=f\left(\psi^{\phi}\right) \circ \phi
$$


(note that $f(\phi)=\phi$ ). But (10) is equivalent to

$$
\mathcal{F}\left(\psi^{\phi}\right)=\phi^{-1} f(\psi) \phi
$$

which is obvious from (8) since conjugation is a group homomorphism, and clearly

$$
\phi^{-1}(\phi(b)) \phi=b
$$

where $b$ is a curve in $X$ (note that we use the same notation for a curve and Dehn twist around it).

Therefore, $\Lambda$ acts on $\mathcal{C}_{\text {top }}^{p}$, so all we need to show is that the action is strictly compatible with disjoint union and gluing. Note that this too is obvious, as those operations are preserved by gluing. (Strictness is made possible by the fact that the action is trivial on objects.)

Now from Theorem 4 we can deduce that $\Lambda$ acts on modular functors. Let us make this quite precise, however. Modular functors with labels $\mathcal{M}$ on any SLCMC $\mathcal{D}$ form a groupoid $\operatorname{Mod}(\mathcal{D}, \mathcal{M})$ because SLCMC's, as any lax versions of any universal algebra, form a 2-category (see the Appendix or Fiore [5] for details). The morphisms of the groupoid $\operatorname{Mod}(\mathcal{D}, \mathcal{M})$ are simply 2-morphisms of that 2-category. Now let $G$ be a group and $S$ be a groupoid. We say that $G$ acts laxly on $S$ if $S$ has the structure of a lax algebra over the theory of $G$-sets (see again [5] for details). We then obtain for formal reasons the following

Corollary 5. The action described in Theorem 4 induces a lax action of the group $\Lambda$ on the groupoid $\operatorname{Mod}\left(\mathcal{C}_{\text {top }}^{p}, \mathcal{M}\right)$ for any set of labels $M$.

Remark: There are numerical invariants attached to modular functors. Thinking of $\mathcal{M}$ as a 2 -vector space, then there is a canonical vector space $\operatorname{tr}(\mathcal{M})$ which, if we view $\mathcal{M}$ as a free 2 -vector space with a basis $A$ (the set of labels), is simply the free $\mathbb{C}$-vector space with basis $A$. Now for a modular functor, $M$ becomes a commutative algebra by letting the product of two labels $a, b$ be simply the sum of all labels $c$ with coefficients given by the dimensions of the value of the modular functor on a pair of pants with inbound boundary components $a, b$ and outbound boundary component $c$. This is the Verlinde algebra. For a modular functor, one may also identify $M$ with its value on an elliptic curve. Now the Verlinde conjecture [21] states that the Verlinde algebra is simply a product of copies of $\mathbb{C}$, and that the splitting is got from the basis $A$ by applying the modular transformation $-1 / \tau$ (thinking of $M$ as the value of the modular functor on the elliptic curve). The Verlinde conjecture has been proved by a number of people using different hypotheses. One of the first outlines of a proof is in [13] (although it needs to be said that a notable hypothesis there is unitarity which is not compatible with $\mathcal{C}^{\text {top }}$, and it seems to be a subtle question if the assumption of unitarity can be replaced by finiteness). Using the machinery of [8], [9], [5] and 
[7] one can, in fact, turn [13] into a rigorous mathematical proof. An alternate approach is in [19], see also [1, 2].

Be it as it may, for a finite modular functor one gets a set of algebraic numbers which are the eigenvalues of the fusion rules of each label, and indeed in examples which can be computed, the absolute Galois group acts on these algebraic numbers in a way compatible with the action given by Corollary 5 . It is an interesting problem to ask how general this action can get. In other words, from the interpretation of $\mathcal{C}_{\text {top }}$ as a Teichmüller tower, and its presumptive universality, one would expect modular functors with fairly wild and general eigenvalues. However, such examples so far do not seem to be known.

Another interesting question is in fact if, and in what sense, can the action of the absolute Galois group of $\mathbb{Q}$ be extended to $\mathcal{C}$. Now to that end, one obviously needs an algebraic (instead of just analytic) version of $\mathcal{C}$. Although there are algebraic approaches to CFT via vertex operator algebras or chiral algebras, an SLCMC approach has so far not been developed. Although it may seem that it is impossible to glue algebraically a curve of higher genus out of curves of lower genus, a kind of such gluing in fact does exist by means of power series rings (generalizing the Tate curve). Nevertheless, a consistent definition of Segal CFT in the algebraic world so far doesn't seem to exist. One remark is in order: in the topolocial world, it is possible to make $\operatorname{Gal}(\mathbb{C} / \mathbb{R})$ act on modular functors, but it reverses their chirality (i.e. takes holomorphic modular functors to antiholomorphisms). Thus, in algebraic CFT, one must have chiralities corresponding a priori to all representations of the absolute Galois group, or at least the cyclotomic character.

\section{Appendix: The Definition of lax COMmutative Monoid With CANCELATION (LCMC)}

The material of this section follows [8], [9], [5]. We omit the treatment of stacks, as it is not directly relevant to this note.

To define lax algebraic structures, we must first define the corresponding strict structures. However to get a hold on the coherence diagrams which will arise in the lax structures, it is helpful to encode strict algebraic structures in some uniform way. The first approach to such definition is the notion of theory according to Lawvere ([11]). Lawvere defines a theory as a category with objects $\mathbb{N}$ such that $n$ is the product of $n$ copies of 1 ; concrete choices of product projections are a part of the structure. A morphism of theories is a functor which strictly preserves the products (i.e. takes $n$ to $n$ and projections to projections). There is also an alternate equivalent definition, which has its own benefits. For a theory 
$T$, we may put

$$
T(n)=\operatorname{Hom}_{T}(n, 1),
$$

and write down the axioms for $T(n)$. Then a theory $T$ is a functor from the category whose objects are natural numbers $\mathbb{N}=\{0,1,2, \ldots\}$ and morphisms from $k$ to $m$ are maps of sets $\{1, \ldots, k\} \rightarrow\{1, \ldots, m\}$ together with a distinguished element $1 \in T(1)$, a composition operation

$$
\gamma: T(k) \times T\left(n_{1}\right) \times \ldots \times T\left(n_{k}\right) \rightarrow T\left(n_{1}+\ldots+n_{k}\right) .
$$

The operation $\gamma$ is associative and unital, and equivariant with respect to the functorial structure, in the obvious sense. For a set $X$, we have the endomorphism theory $\operatorname{End}(X)$ where $\operatorname{End}(X)(n)=\operatorname{Map}\left(X^{\times n}, X\right)$. It is good to note that theories are an example of multisorted universal algebra, which means universal algebra modelled on an indexed set of objects (in this case, $\mathbb{N}$ ). The axioms of such universal algebra are, however, just as in the case of a 1-sorted universal algebra, defined purely in terms of operations and defining relations, which we described. A T-algebra on $X$ is a map of theories

$$
T \rightarrow \operatorname{End}(X) \text {. }
$$

Defining a lax algebra $X$ over a theory $T$ is not difficult. $X$ is required to be a groupoid (more generally a category), and we set

$$
\operatorname{End}_{\text {lax }}(X)=\text { Functors }\left(X^{n}, X\right) \text {. }
$$

Then a structure of a lax T-algebra on $X$ is given by a map

$$
\phi: T \rightarrow \operatorname{End}_{l a x}(X)
$$

and natural isomorphisms

$$
\begin{gathered}
\phi(1) \cong I d, \\
\phi\left(\gamma\left(g ; g_{1}, \ldots, g_{k}\right)\right) \cong \gamma\left(\phi(g) ; \phi\left(g_{1}\right), \ldots, \phi\left(g_{k}\right)\right), \\
\phi\left((T(f)(g)) \cong\left(\operatorname{End}_{l a x}(X)(f)\right)(\phi(g)) .\right.
\end{gathered}
$$

These are called coherence isomorphisms of the lax algebra $X$. It is important to notice that the coherence isomorphisms correspond to operations of the universal $\mathbb{N}$-sorted algebra defining theories (i.e. unit, composition and functoriality). To complete the definition of lax $T$-algebra, the coherence isomorphisms are subject to coherence diagrams which are required to commute. These diagrams now correspond in an obvious way to relations in the $\mathbb{N}$-sorted universal algebra describing theories, i.e. the associativity, unit and equivariance relations mentioned above. Therefore, coherence diagrams can be easily written for any lax universal algebra as a matter of general principle, which is not at all obvious how to do without introducing theories. For completeness, we point out that a lax morphism of lax algebras $X, Y$ over a theory is defined as a functor

$$
F: X \rightarrow Y
$$


For $f \in T(n)$, a part of the structure of $F$ are coherence isomorphisms

$$
f(F, \ldots, F) \cong F f .
$$

These are required to satisfy commutative coherence diagrams which however now are indexed by the operations (not relations) of the $\mathbb{N}$-sorted universal algebra defining theories.

Lax $T$-algebras further form a 2-category. There are various possible choices of the 2-morphisms, among which for our purposes the suitable choice is simply to take a 2-morphism $F \rightarrow G$ to be a natural isomorphism

$$
\phi: F \rightarrow G
$$

which commutes with the coherence isomorphisms of $F, G$.

Unfortunately, this framework of lax $T$-algebras, while useful, is not sufficient to encode a structure such as $\mathcal{C}_{\text {top }}$. The point is that the operation of gluing is indexed by inbound and outbound boundary components, which vary depending on the element (e.g. rigged surface) to which the operation is being applied! To capture this, we have introduced a notion of 2-theory $\Theta$ fibered over a theory $T$. (Caution: some authors use the term 2-theory to denote merely the analogue of theory in the realm of 2-categories. That is different from the present notion.)

A 2-theory consists of a natural number $k$, a theory $T$ and a (strict) contravariant functor $\Theta$ from $T$ to the category of categories (and functors) with the following properties. Let $T^{k}$ be a category with the same objects as $T$, and $H_{o m} T^{k}(m, n)=H_{T}(m, n)^{\times k}$. Then

$$
\operatorname{Obj}(\Theta(m))=\coprod_{n} \operatorname{Hom}_{T^{k}}(m, n),
$$

for $\phi: m \rightarrow n$ in $T$, the map $\operatorname{Obj}(\Theta(n)) \rightarrow \operatorname{Obj}(\Theta(m))$ which is a part of $\Theta(\phi)$ is given by precomposition with $(\phi, \ldots, \phi)$ and

$$
\gamma \in \operatorname{Hom}_{T^{k}}(m, n)
$$

is the product, in $\Theta(m)$, of the $n$-tuple

$$
\gamma_{1}, \ldots, \gamma_{n} \in \operatorname{Hom}_{T^{k}}(m, 1)
$$

with which it is identified by the fact that $T$ is a theory. (We need to allow $k>1$ because in the example we are interested in, $k=2$.)

Again, one can write down the axioms of a 2-theory explicitly, by letting

$$
\Theta\left(w ; w_{1}, \ldots, w_{n}\right)=\operatorname{Hom}_{\Theta(m)}\left(\left(w_{1}, \ldots, w_{n}\right), w\right)
$$


for $w_{i}, w \in T(m)^{\times k}$ where the $n$-tuple $\left(w_{1}, \ldots, w_{n}\right)$ is identified with the corresponding element of $\mathrm{Hom}_{T^{k}}(m, n)$.

Let $T$ be a theory, and let $k \in \mathbb{N}$ be a fixed number. Then, as remarked, for a number $m \in \mathbb{N}$ and for any elements

$$
w_{1}, \ldots, w_{n}, w \in T(m)^{\times k}
$$

we have a set

$$
\Theta\left(w ; w_{1}, \ldots, w_{n}\right)
$$

There are the following operations on a 2-theory:

(1) A unit $1 \in \Theta(w ; w)$ where $w \in T(m)^{\times k}$ is any element.

(2) $\Theta$-composition

$\gamma: \Theta\left(w ; w_{1}, \ldots, w_{n}\right) \times \Theta\left(w_{1} ; w_{11}, \ldots w_{1 p_{1}}\right) \times \ldots \times \Theta\left(w_{n} ; w_{n 1}, \ldots w_{n p_{n}}\right) \rightarrow \Theta\left(w ; w_{11}, \ldots w_{n p_{n}}\right)$

where all $w, w_{i}, w_{i j} \in T(m)^{\times k}$.

(3) $\Theta$-functoriality: for a map

$$
\iota:\{1, \ldots, q\} \rightarrow\{1, \ldots n\},
$$

a map

$$
\Theta\left(w ; w_{\iota(1)}, \ldots, w_{\iota(q)}\right) \rightarrow \Theta\left(w ; w_{1}, \ldots, w_{n}\right) .
$$

(4) $T$-functoriality: for a map

$$
\iota:\{1, \ldots, m\} \rightarrow\{1, \ldots, q\}
$$

a map

$$
\Theta\left(w ; w_{1}, \ldots w_{n}\right) \rightarrow \Theta\left(\iota^{\times k} w ; \iota^{\times k} w_{1}, \ldots \iota^{\times k} w_{n}\right) .
$$

(5) $T$-substitution: For $u_{i} \in T\left(k_{i}\right), i=1, \ldots, m$, and $v_{i}=\gamma^{\times k}\left(w_{i} ; u_{1}^{\times k}, \ldots u_{m}^{\times k}\right)$, $v=\gamma^{\times k}\left(w ; u_{1}^{\times k}, \ldots, u_{m}^{\times k}\right)$, a map

$$
\Theta\left(w ; w_{1}, \ldots, w_{n}\right) \rightarrow \Theta\left(v ; v_{1}, \ldots, v_{n}\right) .
$$

The axioms (relations) required of a 2-theory are: associativity and unitality of $\Theta$-composition, associativity of $\Theta$-functoriality, $\Theta$-equivariance, associativity of $T$-functoriality and $T$-substitution, $T$-equivariance, and commutativity between $T$-substitution and $T$-functoriality and $\Theta$-composition and $\Theta$-functoriality. The meaning of these axioms is clear, and will not be given in detail here (since one can always use the categorical definition to write them down). Similarly, it is clear what one means by (strict) morphism

$$
(\Theta, T) \rightarrow(\Sigma, S)
$$

where $\Theta$ is a 2-theory fibered over $T$ and $\Sigma$ is a 2-theory fibered over $S$.

Now consider a set $I$ and a map

$$
X: I^{k} \rightarrow \text { Sets. }
$$


To such data there is assigned a 2-theory $\operatorname{End}(X)$ fibered over the theory $\operatorname{End}(I)$ : let

$$
\Theta\left(w ; w_{1}, \ldots, w_{n}\right)
$$

consist of the set of all possible simultaneous choices of maps

$$
X\left(w_{1}\left(i_{1}, \ldots, i_{m}\right)\right) \times \ldots \times X\left(w_{n}\left(i_{1}, \ldots, i_{m}\right)\right) \rightarrow X\left(w\left(i_{1}, \ldots, i_{m}\right)\right)
$$

where $i_{j}$ range over elements of $I$. A structure of an algebra over the 2-theory $\Theta$ fibered over $T$ is given by a morphism

$$
(\Theta, T) \rightarrow(\operatorname{End}(X), \operatorname{End}(I)) .
$$

Example: The example relevant to $\mathcal{C}_{\text {top }}$ is the 2-theory of commutative monoids with cancellation (CMC): $T$ is the theory of commutative monoids with an operation + . We set $k=2$. The 2-theory $\Theta$ has three generating operations, addition (or disjoint union)

$$
+: X_{a, c} \times X_{b, d} \rightarrow X_{a+b, c+d},
$$

unit

$$
0 \in X_{0,0}
$$

and cancellation (or gluing)

$$
\check{?}: X_{a+c, b+c} \rightarrow X_{a, b} .
$$

(In this notation, $X$ is a general CMC, i.e. algebra over the 2-theory of CMC's.) The axioms are commutativity, associativity and unitality for,+ 0 , transitivity for ?

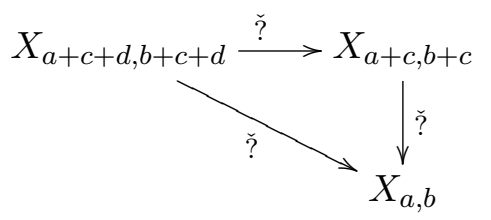

(note that the cancellation operation of $c+d$ uses $T$-substitution) and distribu-

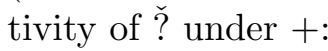

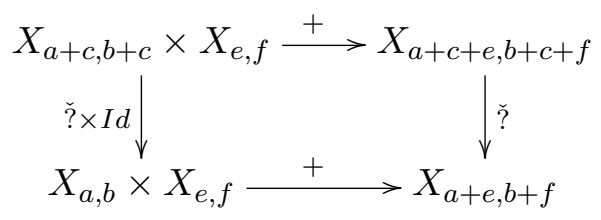

(similarly, note that in this diagram, $T$-substitution is used).

However, $\mathcal{C}$ or $\mathcal{C}_{\text {top }}$ are, as pointed out, not CMC's, but rather lax algebras over that theory (LCMC's). 
To define a lax algebra over $(\Theta, T)$, let $I$ be a groupoid (or more generally category), and let $X$ be a strict functor from $I$ to groupoids. We have already defined $\operatorname{End}_{l a x}(I)$. To define

$$
\operatorname{End}_{l a x}(X)\left(w ; w_{1}, \ldots, w_{n}\right),
$$

we take the set of simultaneous choices of functors (12) for each $\left(i_{j}\right)_{j} \in I^{m}$, which are strictly natural transformations (where $X\left(w_{i}\left(i_{1}, \ldots, i_{m}\right)\right)$ is a functor in $I^{m}$ using the strict functoriality of $X$, and the usual functoriality of Hom's).

Now a lax algebra over $(\Theta, T)$ consists of a lax algebra $I$ over $T$ (i.e. in particular a map $\left.\phi: T \rightarrow \operatorname{End}_{l a x}(I)\right)$, a strict functor $X$ from $I^{k}$ to groupoids, and a map

$$
\Theta\left(w ; w_{1}, \ldots, w_{n}\right) \rightarrow \operatorname{End}_{l a x}(X)\left(\phi(w) ; \phi\left(w_{1}\right), \ldots, \phi\left(w_{n}\right)\right)
$$

together with a natural coherence isomorphism for each operation $1, \ldots, 5$ of a 2-theory, and a commutative coherence diagram for each relation among the operations of a general 2-theory (see above for the list of such relations). To clarify this, note that 2-theories $\Theta$ fibered over a fixed theory $T$ in effect again are the same as algebras over a multisorted universal algebra characterized by the operations $1, \ldots, 5$ and the corresponding relations. Similarly as above, now coherence isomorphisms for an algebra over a 2-theory correspond to the operations of that multisorted universal algebra, and coherence diagrams correspond to its relations.

Now it is easy to show (although messy to write down) that $\mathcal{C}, \mathcal{C}_{\text {top }}$ together with any reasonable definition of gluing and disjoint union indeed is a lax commutative monoid with cancellation (LCMC). Note that the benefit of a fixed framework is enormously increased when passing from theories to 2-theories. Even writing down what coherence diagrams should be present as a matter of general principle for an LCMC is a rather complex task. It is, of course, noteworthy that Moore-Seiberg [13] were nevertheless able to work with these coherences intuitively.

Note that, similarly as above, lax algebras over a 2-theory $\Theta$ in this sense form a 2-category similarly as lax algebras over a theory. Again, different choices of 2morphisms are possible, but for the purposes of this paper, it suffices to consider the case when the underlying $T$-algebra $I$ is fixed and the underlying morphism on $I$ is the identity (as it is always the lax commutative monoid of sets with the operation of disjoint union). Then, we can define again such lax map between two $\Theta$-algebras represented by strict functors $X, Y: I^{k} \rightarrow$ Groupoids to be a natural transformaton $\phi: X \rightarrow Y$ together with natural coherence isomorphisms, for $f \in \Theta\left(w ; w_{1}, \ldots, w_{n}\right)$,

$$
\phi f\left(w(i) ; w_{1}(i), \ldots, w_{n}(i)\right) \cong f\left(w(i) ; w_{1}(i), \ldots, w_{n}(i)\right)(\phi, \ldots, \phi)
$$


for $i \in I^{k}$, which satisfy commutative coherence diagrams indexed by the $o p$ erations of the multisorted universal algebra describing 2-theories fibered over $T$.

\section{REFERENCES}

[1] B.Bakalov, A.Kirillov: Lectures on tensor categories and modular functors, University Lecture Series 21, Amer. Math. Soc., Providence, RI, 2001

[2] B.Bakalov, A.Kirillov: On the Lego-Teichmüller game, Transformation Groups 5 (2000) 3 , 207-244

[3] P. Degiovanni: Moore and Seiberg equations, topological field theories and Galois theory, The Grothendieck theory of dessins d'enfants, L. Schneps, ed. London Math. Soc. Lecture Note Series 200 (1994), 359-368

[4] V. G. Drinfeld; On quasitriangular quasi-Hopf algebras and a group closely connected with $\operatorname{Gal}(\overline{\mathcal{Q}} / \mathcal{Q})$, Leningrad Math. J. 2 (1991), 829-860

[5] T. Fiore: Pseudo limits, Bi-Adjoints, and Pseudo Algebras: Categorical Foundations of Conformal Field Theory, CT/0408298

[6] A. Grothendieck: Esquisse d'un programme, in Geometric Galois Actions, L.Schneps and P. Lochak eds., London Math. Soc. Lecture Notes 242, Cambridge University Press, 1997

[7] A. Hatcher, P. Lochak and L. Schneps: On the Teichmüller tower of mapping class groups, J. Reine Angew. Math. 521 (2000) 1-24

[8] P. Hu, I. Kriz: Conformal field theory and elliptic cohomology, Advances in math. 189 (2004) 325-412

[9] P. Hu, I. Kriz: Closed and open conformal field theories and their anomalies, Comm. Math. Physics 254 (2005) 221-253

[10] I. Kriz: On spin and modularity in conformal field theory, Annals Sci. École Norm. Sup. (4) 36 (2003) 51-112

[11] W.F.Lawvere: Functorial semantics of algebraic theories, Proc. Nat. Acad. Sci. U.S.A. 50 $1963869-872$

[12] P. Lochak, H. Nakamura, L. Schneps: On a new version of the Grothendieck-Teichmüller tower, C. R. Acad. Sci. Paris 325, Ser. 1, (1997) 11-16

[13] G. Moore, N. Seiberg: Polynomial equations for rational conformal field theories, Phys. Lett. B 212 (1988) 451-460

[14] H. Nakamura, L. Schneps: On a subgroup of the Grothendieck-Teichmüller group acting on the tower of profinite Teichmüller modular groups, Inventiones Math. 141 (2000) 503-560

[15] D. Quillen: Determinants of Cauchy-Riemann operators on Riemann surfaces Funktsional. Anal. i Prilozhen. 19 (1985) 37-41

[16] L. Schneps: Fundamental groupoids of genus zero moduli spaces and braided tensor categories, In Moduli spaces of curves, mapping class groups and field theory.

[17] L. Schneps: Automorphisms of curves and their role in Grothendieck-Teichmüller theory, preprint, 2004

[18] L. Schneps: Lecture II: Curve Complexes, Tensor Categories, Fundamental Groupoids, http://www.aimath.org/WWN/motivesdessins/schneps2.pdf

[19] G. Segal: The definition of conformal field theory, preprint in the 1980's, now published in Topology, geometry and quantum field theory, 421-577, London Math. Soc. Lecture Ser. 308, Cambridge Univ. Press, Cambridge, 2004

[20] V.G. Turaev: Quantum invariants of knots and 3-manifolds, de Gruyter Studies in Mathematics 18, Walter de Gruyter and Co., 1994 
[21] E. Verlinde: Fusion rules and modular transformations in 2-D conformal field theory, $\mathrm{Nu}$ clear Phys. B 300 (1988) 360-376

\section{$\mathrm{Po} \mathrm{Hu}$}

Wayne State University

Department of Mathematics

Faculty Administration Building Detroit, MI 48202

E-mail:po@math.wayne.edu

\section{Igor Kriz}

Department of Mathematics

University of Michigan

E-mail:ikriz@umich.edu 